Review began 09/18/2021 Review ended 09/23/2021 Published 09/27/2021 Retracted 11/18/2021 Corrected 11/09/2021

(c) Copyright 2021

Farhan Y Almalki et al. This is an open access article distributed under the terms of the Creative Commons Attribution License CC-BY 4.0., which permits unrestricted use, distribution, and reproduction in any medium, provided the original author and source are credited.

\section{Retracted: The Role of Heat Shock Proteins in Cellular Homeostasis and Cell Survival}

\author{
Abdullah Farhan Y Almalki ${ }^{1}$, Maria Arabdin ${ }^{2}$, Adnan Khan ${ }^{3}$ \\ 1. Pathology, University of Malta, Msida, MLT 2. Pathology, Rehman Medical College, Peshawar, PAK 3. Pediatrics, \\ Rehman Medical Institute, Peshawar, PAK \\ Corresponding author: Adnan Khan, adnan-khan@outlook.com
}

\section{This article has been retracted.}

Retraction date: November 18, 2021. Cite this retraction as Farhan Y Almalki A, Arabdin M, Khan A (November 18, 2021) Retraction: The Role of Heat Shock Proteins in Cellular Homeostasis and Cell Survival. Cureus 13(11): r36. doi:10.7759/cureus.r36.

It has come to our attention that figures 1 and 3 in this article have been reproduced by the authors without first obtaining permission from the original publishers or authors:

Figure 1: Akerfelt M, Morimoto RI, Sistonen L: Heat shock factors: integrators of cell stress, development and lifespan. Nat Rev Mol Cell Biol. 2010, 11:545-55. 10.1038/nrm2938

Figure 3: Pockley AG. Heat shock proteins in health and disease: therapeutic targets or therapeutic agents? Expert Rev Mol Med. 2001, 3:1-21. 10.1017/S1462399401003556.

In addition, Figure 3 was also not properly attributed to the original source. This was later fixed via correction. After confirming with the publisher of Figure 1 that permission to republish was not obtained, and receiving no response from the authors despite multiple attempts, we have made the decision to retract this article and completely remove Figures 1 and 3 from the online and PDF versions of the article.

\section{This article has been corrected.}

Correction date: November 09, 2021. Cite this correction as Farhan Y Almalki A, Arabdin M, Khan A (November 09, 2021) Correction: The Role of Heat Shock Proteins in Cellular Homeostasis and Cell Survival. Cureus 13(11): c52. doi:10.7759/cureus.c52.

Figure 3 was originally published in Expert Reviews in Molecular Medicine (2001) Cambridge University Press. This information was mistakenly deleted from the figure legend during revisions and has now been readded to the Figure 3 legend. Cureus regrets the error.

\begin{abstract}
This review article has been necessitated by the limited number of studies on the role of heat shock proteins (HSPs) in cellular functions. The analysis is performed by reviewing evidence in various literary works concerning the topic. The main function of HSPs is to prevent the formation of non-functional proteins and facilitate protein folding. They also enhance the survival of cells in addition to being clinically significant. HSPs protect proteins from stress factors such as temperature, $\mathrm{pH}$, and low levels of oxygen. Some of the common types of HSPs include HSP70, HSP90, HSP27, and HSP100. These proteins have different weights and other features which make them suit for different cellular functions. However, they have numerous similar features which make them perform almost the same functions, yet they vary in the degree of protection that they provide for the cells. The release of HSPs is controlled by four types of HSF depending on the type of stress that a cell is subjected to. HSF1 is responsible for identifying stress factors, especially heat. HSF2 performs almost similar functions as HSF1 in addition to cellular development. HSF3 is released when the stress conditions are extreme and, hence, cannot be effectively controlled by HSF1 and HSF2. HSF4 functions by inducing negative DNA transcriptions. Other tasks of HSPs include enhancing the immune system. The cells help in the management of Alzheimer's disease and other similar complications by forming protective tissues around brain cells. The cells also help in controlling cancer and heart diseases. However, their roles are more enhanced in managing cancer, extending to diagnosis and prediction. Further research on the HSPs and HSFs may extend their application to curing tumorous cells.
\end{abstract}

Categories: Genetics, Pathology, Hematology 


\section{Introduction And Background}

Heat shock proteins (HSPs), also known as stress proteins, are a collection of effective proteins involved in a variety of different range of important cellular functions and can be found in both eukaryotic and prokaryotic cells. In protein folding, protein chains are reconfigured into three-dimensional shapes in the endoplasmic reticulum for them to function properly. However, misfolding of the protein chains may occur for three major reasons. Firstly, mutation may make certain proteins lack the preferred folds. Secondly, ribosomes may erroneously decode DNA information which should be applied in the correct folding process. Thirdly, it may be hard for the protein to completely fold correctly even if there are neither mutation nor decoding challenges. For that reason, HSPs play a significant role in protein folding process and elimination of misfolded proteins [1]. Additionally, they involve in the signaling pathways and regulation of the immune system. This is an important physiological process enabling a group of molecules to work together in controlling cell functioning. This is attained through the activation of the protein kinase which is vital in regulating the functioning of other proteins through phosphorylation. HSPs have a unique functional property that allows them to be essential not only in protein regulation but also in cell survival and development. A large number of chaperones are considered as HSP, and their primary function is to prevent the formation of nonfunctional proteins and mediate protein folding [2]. The level of these proteins increases significantly in response to proteins denaturation. Proteins miss-folding and denaturation occur as a result of different types of stress such as high temperature, $\mathrm{pH}$, and low $\mathrm{O}_{2}$ level. Essentially, the performance of most of the proteins is optimal at the body temperature of about $37^{\circ} \mathrm{C}$. At temperature levels above $41^{\circ} \mathrm{C}$, correct functioning of the proteins is hampered, and a further increase leads to destruction called denaturation. On the other hand, maintaining the optimal $\mathrm{pH}$ level is significant for proteins since inappropriate acidity or alkalinity can affect solubility and electrolytic charges. In this concern, the normal $\mathrm{pH}$ range for the right functioning of the proteins is 4.5-7.4. HSPs also repair the damages caused to the body suffering from such harsh conditions. This, therefore, means that they retain the homeostasis of proteins in a body during various cellular functions, a function made possible by their vast adaptability [2]. Depending on their locations within a cell, their functions are varied. Response to elevated heat by organisms is achieved through thermotolerance as well as acclimatization. HSPs are very critical in the thermotolerance mechanism. In this case, prior exposure to extreme heat becomes a stimulus that triggers the accumulation of HSPs, which can subsequently protect functional proteins from being denatured. Contrastingly, acclimatization entails the adaption of the organisms to retain a certain level of activeness despite an increase in temperature. In this regard, it has been established that HSPs are useful in enabling some physical responses like the transfer of heat to the skin surface or increasing sweating output. Heat shock proteins are categorized into small families which are further divided into smaller sub-families depending on their molecular weight [3]. Particularly, the sizes of the HSPs range from 10 to 100 kilo Dalton (kDa). In this regard, they have been generally classified into small and large proteins. Based on the functions, HSPs are broadly divided into those regulating chaperonin, signal transduction, and ribonucleic acid (RNA) activities. The physical points at which the HSPs are found inside the cells also play some important roles in the categorization. Notably, the primary locations containing the HSPs are the mitochondria, cytoplasm, cytosol, plasma membrane, and the endoplasmic reticulum. However, in this paper, some of the major families will be discussed in this review. The main emphasis of this review will be on the small heat-shock proteins (sHsps), HSP70, HSP90, HSP27 and HSP100 families, their regulatory role in cellular functions, survival and clinical significance.

\section{Review}

\section{HSP70}

This is the most common of the HSP family. The member proteins find expression in the cell as they play a critical role not only in preventing protein aggregation but also in facilitating the folding of proteins and protecting the skin from both thermal and oxidative stress. The three types of proteins found in prokaryotes are DnaK, HscA, and HscC. Eukaryotic organisms have a different set of HSP70. The general characteristic of HSP70 proteins is that its genetic setup is made up of 3 functional elements that include the $\mathrm{N}$-terminal ATPase, the substrate-binding domain, and the C-terminal domain. HSP70 proteins in their natural state are bound in the form of ATP. The interaction of HSP70 family member proteins with peptides is crucial in regulating cell activity [4].

\section{HSP27}

HSP27 is a major HSP can be found in both prokaryotes and eukaryotic. It is an ATP-independent protein, present in normal and abnormal tissues. HSP in human beings is genetically passed on by the HSPB1 gene. It is located in chromosome 7 in human beings. HSP27 is a chaperone of a small HSP. The cell structure of HSP27 consists majorly of a cell nucleus and a cytoskeleton. It is essential in multiple molecular processes. These include RNA binding, ubiquitin-binding and protein binding [5].

Some of its biological functions include retina homeostasis, positive regulation of angiogenesis, platelet aggression and movement of the cellular and sub-cellular components. However, the most important function of HSP27 is thermos-tolerance and aid in the cells during stressful conditions. HSP27 does this 
through the lowering of reactive oxygen species. This is made possible by increasing glutathione and decreasing the levels of iron in the cells. HSP27 interaction with actin prevents the actin's filaments from fragmentation. As a chaperone, it joins together misfolded proteins in ATP transferring. It also aids in the refolding of denatured proteins to form active conformations. Therefore, HSP27 plays a pivotal role as a possible biomarker and therapeutic target [6].

\section{HSP90}

Just like HSP27, the HSP90, is a chaperone that assists other proteins into folding well. Like most HSPs, it equally helps in stabilizing proteins from heat stress and also in the degradation of proteins. HSP90 is ATPdependent and is vital in eukaryotes. Among the many physiological processes that depend on this HSP include the following: transport within the cell structure, protein degradation and signal transduction. As a result, HSP90 has become very essential in cancer-related therapies. When faced with thermal stress, HSP90 is capable of preventing the denaturation of proteins. Through a cytosolic complex, it also promotes the refolding of denatured proteins [7].

\section{HSP100}

Hsp100 proteins family found in prokaryotic and eukaryotic organisms and can be further classified according to the abundance of AAA domains into two subtypes, a group with two nucleotide-binding sites and second with only one nucleotide-binding site. Some HSP100 are involved in the destruction of tagged and misfolded proteins, and some act as chaperones [8].

\section{Small HSPs}

sHSP are functional proteins act as chaperones preventing stress from inducing protein denaturation, blocking protein aggregation and improving cellular survival during extreme condition. SHSP also has the ability to isolate and store unfolded proteins. sHSP is present primarily in the cytoplasm and nucleus [9]. Figure 1 shows the functions of HSPs during extreme conditions [10].

\section{FIGURE 1 REMOVED DUE TO RETRACTION}

\section{Regulation of HSPs}

The expression of HSP depends on the transcription factors HSF. The HSF regulates the release of HSP depending on the nature of the stressors. In essence, our body has gone through tremendous exposure to stress over time prompting the cells to survive through adaptation. Therefore, the role of HSF comes out as inducer to certain HSP necessary for sequestering an impending stressing condition in the cells [11]. The HSF remains dominant in the nucleus and cytoplasm in a monomeric form waiting to be activated by "heat shock response" (HSR). Following activation, HSF trimerizes and moves to the nucleus, where they bind to the promoter region of HSP genes inducing their activation, leading to a significant increase in HSP expression. Our body tissues face a large amount of stressful exposure that can result in a chronic or mild strain. Therefore, the cells have gone through significant evolutions to ensure that they can adapt, predict, and overcome impending external and internal stress [11]. The adaptation of the cells has sharpened the capability of HSPs regulation and reaction whenever they are required in the body. The expression of heat shock gene depends on the activation of transcription factor (HSF). In this case, the changes in the environment influence the activity of HSF that in turn causes the assemblage of HSPs. HSF can be divided into 4 groups, HSF1, 2, 3, and 4, and depending on the type of shock, HSF induces HSP [12]. HSFs have four distinct domains that include " $\mathrm{N}$-terminal, an oligomerization domain and trans-activation domain" that work together in reacting to different stresses. The $\mathrm{N}$-terminal recognizes the DNA sequences whereas the oligomerization domain is responsible for forming the trimer shape of the protein. The C-terminal has the mandate of regulating stability in the cell by detecting heat or other stressors [13]. Figure 2 shows an overview of the HSF family members and their functions [13]. 


\section{Cureus}

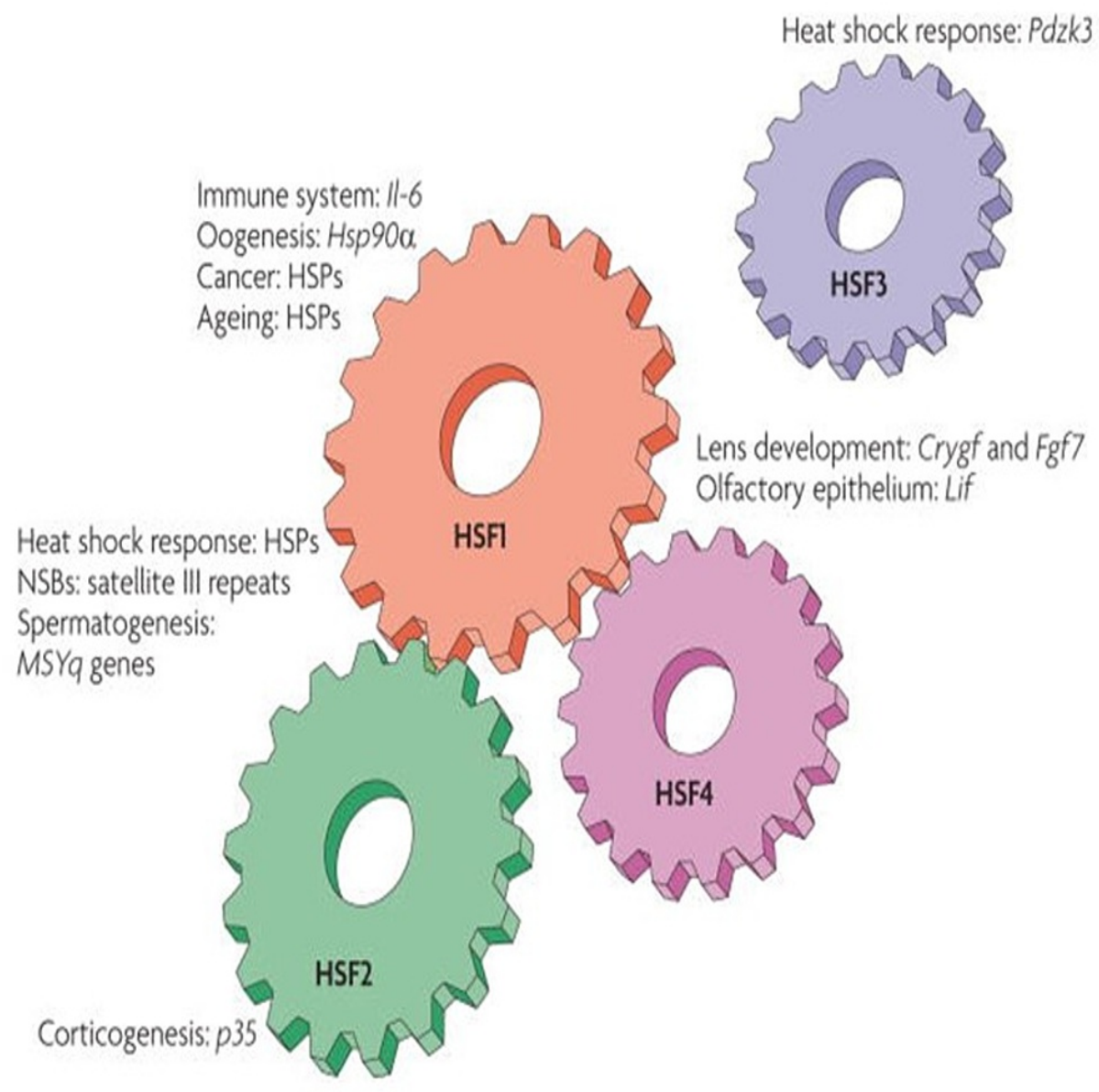

FIGURE 1: Overview of the HSF family members and their functions.

HSPs: heat shock proteins.

\section{HSF1}

It is important to understand the activities of 'Heat shock factor 1' (HSF1) in inducing the production of HSP, HSF1 has a crucial role in identifying stressors and triggering the action of HSPs. In this case, we can see their critical role in capturing heat stress even when the cells have not been affected [14]. The location of HSF1 is fundamental due to their innate functions in the cell. Therefore, they are located in the outer cell membrane to allow for easy detection of stress.

Consequently, the HSF1 shows unique abilities of 'transactivating genes' making it essential in the activation of HSPs. The functions of HSF1 complements the inherent role of HSP of reacting to stressors in the cell. However, HSF1 has other roles that include gametogenesis, metabolism, as well as aging [11].

It is important to understand the different genes that affect the expression of HSPs through inductions by HSFs. A study has shown that HSF1 can be triggered by a multitude of stressors such as "oxidative stress, heat, and amino acid imbalances." Here, it is apparent that HSF trimer formation cannot rely on temperature ranges but instead the cell can use other biochemical factors to detect stress. These factors can include changes in $\mathrm{pH}$. that indicates growth of certain microorganisms such as bacteria in the cells. Therefore, the biochemical composition of the HSFs remains crucial in its quest to initiate transcription of an impending stress in the tissues [15].

\section{HSF2}

HSF2 plays a supportive role with HSF1 during the stressful condition, adding their role in cellular development and differentiation. Unlike HSF1 the way HSF2 get activated differs, as particular inhibitors of protein degradation processes are required in order to induce their activation. However, HSF2 during stressful conditions induces the same HSPs as HSF1 [12]. 
We see the presence of HSF3 that remains evident in avian and later on identified in mice. During transcription, HSF3 can be triggered by HSF1. This occurs due to the extreme conditions of stress forcing HSF1 to initiate the up-regulation of HSF3. These diverse roles make HSF1 a significant molecule in the subsequent biological studies that will help solve challenges such as cancer [16].

\section{HSF4}

HSF4 lacks carboxyl-terminal hydrophobic that is seen in the other HSFs. This makes HSF4 qualified for inducing negative DNA transcriptions. HSF4 expression is limited to only a few tissues. HSF4 has an important role in the development and maintaining of the lens [17].

Figure 3 shows the regulation of transcription of HSP genes by HSF.

FIGURE 3 REMOVED DUE TO RETRACTION

\section{Role of HSP as molecular chaperones during stress conditions}

Molecules such as proteins are important parts of the body. Molecular chaperones commonly referred to as HSPs are components of living cells that play roles such as maintenance of protein homeostasis. Classification of chaperones is typically in accordance with cell origin where they play central function, in a precise location, and mobility. HSPs that include HSPBs and HSPH family members partly bind to facilitate the repair of damaged proteins due to potential production in stressed cells in an ATP manner that influences the prevention of permanent aggregation against cells and with cell compositions such as a cellular membrane. HSPBs transfer misfolded proteins to ATP-dependent chaperones that help in refolding. Association of HSPA1 with co-chaperones aids in the degradation of damaged proteins hence chaperone system and protein degradation system restricts apoptosis that results in enzymes activation. The occurrence of changes in human malignancy affects the protein folding ability in the conversion from weak to stringent regulation [18].

\section{Role of HSP in membrane quality control}

Nuclear pores enable the movement of proteins to the inner nuclear membrane from the endoplasmic reticulum in the quality-controlled mechanism that enables elimination of misfolded proteins by retranslation to then cytoplasm followed by degradation. HSPs normally binds to lipid membrane with subsequent physical order increase and membrane stabilization during strain situations. Additionally, molecular chaperone binds with lipid membrane in the lipid phase stabilization hence a role in the protein protection activity. It is notable that heat shock protein-membrane association alters stress condition effects in the role of cellular stress management [19]. According to Balogh et al. [20] disorders such as neurodegenerative diseases result from alterations in physical state and lipid cellular membrane composition and heat shock proteins that cause a deficiency in the chaperone gene expression. Intercalating compounds are corrective measures in membrane properties alterations by normalizing deregulated HSP expression hence the reversal of the uncontrolled effect of illness [20].

\section{Role of HSP during oxidative stress}

Elevated oxidative stress has been identified in several diseases like cancer and neurodegenerative disease. Interestingly, some studies have shown that HSPs are involved in protective mechanisms against oxidative stress. This is done by initiating a protective mechanism that allows an increase in cell resistance to the oxidative strain presented, besides correlating with a decrease in levels of hydroxyl radical and iron generation hence low levels of oxidized proteins. For example, some members of sHSP have the ability to reduce the number of reactive oxygen species (ROS), and also influence the regulation of intracellular redox protecting cellular cytoskeleton [10].

\section{Role of HSP in programmed cell death}

Programmed cell death, also called apoptosis is a fundamental process in cellular organisms in order to develop good quality control by the elimination of non-functional cells. This is important for embryonic development, maintenance of tissue homeostasis, and cell viability regulation by hormone and growth factors. During apoptosis intrinsic activity, the release of pro-apoptotic proteins such as cytochromes, to the cytoplasm. This is because of changes in the inner mitochondrial membrane which therefore bind with the enzymes, with activation leading to the initiation of the apoptotic protease cascade, besides some HSPs which hinder various stages of the process. For example, HSPB1 acts in the cell protection against apoptotic inducements in an effect dependent on its oligomer status. In addition, the formation of a cytosolic complex with Apaf-1 influences HSPC activity in the formation prevention of apoptosome [10].

\section{HSP in malignancy}

There are many causes of cancer that have been highlighted in previous years. This is after scientists analyzed some of the factors that might be leading to this deadly disease. HSPs are one of the causes of cancer. Research shows that these proteins play a crucial role in the formation of cells that result in the 
growth of a tumor. This is one of the signs that are common in the early stages of cancer. The number of HSPs is one of the indicators of cancer. A significant increase in the level of these proteins shows the presence of cancer. A high number of HSPs causes malignant cells. This is because they act as protection against apoptosis. The cause could be as a result of treatment or malignancy [10].

Due to the advancements in the use of technology in the medical sector. HSPs are used in the analysis of the various types of cancer. It is useful in identifying the features that are common in this health condition, for instance, the level of differentiation and the type of tumor. For instance, an increase in the number of HSPs shows the development of cancer in some cells. This is considered an abnormal body change that should be dealt with promptly [21]. A good example is high levels of HSP27 shows the presence of hyperplastic endometrium. Besides, an increase in the number of HSP70 is an indication of oral cancer. In other cases, for instance, the cancer of the colon and cervix is identified when there is a high level of HSP60 and HSP10 respectively [22]

The presence and number of HSPs help in showing the grade of cell differentiation. HSPs are used in evaluating the stages of cancers. This is because it is easy to study and analyze these proteins. Keratinocyte differentiation is identified when there is presence of HSP27 in the cells. HSPs are also accompanied with molecules that have a close relationship to cancer formation. For instance, Oestrogen receptor alpha (ER $\alpha)$ is one of the molecules that is accompanied with HSP27 in breast cancer, which is typical among females. Cervical, uterine carcinomas and hepatocellular carcinomas are other tissues that experience these alpha cells during the growth of cancer. Regulation of HSP27 is one of the measures taken to control breast cancer $[22,23]$.

\section{Conclusions}

The knowledge of HSPs and HSFs has remained limited making its application skewed. HSP27, HSP70, HSP90, as well as HSP100, are common and they largely differ in size, function, and their location in the cell. On the other hand, HSF1, HSF2, HSF3, and HSF4 are the most important chaperones or stress proteins that regulate the functioning of HSPs. Accordingly, the adaptability and diversity of the HSPs are highly notable. Though the HSPs are composed of proteins, their primary function entails the protection of other proteins against wide-ranging stressors like heat, oxygen, and PH changes. We have seen that HSPs play a critical role in the refolding, breakdown, and protection of the proteins. They have different molecular weight resulting in diverse categories that tackle the unique challenge. More importantly, varying sizes of the HSPs enable them to occupy different parts of the cells and perform various functions. Some of the HSFs stand out as essential elements in the management of chronic diseases such as cancer and heart conditions. As far as malignancy is concerned, HSPs appear to have roles of proliferating the growth of cancerous cells. On the other hand, it may be applied as a vaccine to inhibit the growth of tumors. When responding to heat shock, these proteins assemble in large numbers hence bringing other beneficial effects to the cell. The facilitation of thermotolerance by the HSPs has been widely studied. However, their heat acclimatization mechanism is yet to be fully understood by most researchers. The detrimental effects of tumorous cells can be averted by the modulation of HSPs. Therefore, we can enhance the assembly of HSPs in infected cells to hasten their recovery. Further study of HSPs and HSF will offer new avenues for applying these proteins in disease control.

\section{Additional Information \\ Disclosures}

Conflicts of interest: In compliance with the ICMJE uniform disclosure form, all authors declare the following: Payment/services info: All authors have declared that no financial support was received from any organization for the submitted work. Financial relationships: All authors have declared that they have no financial relationships at present or within the previous three years with any organizations that might have an interest in the submitted work. Other relationships: All authors have declared that there are no other relationships or activities that could appear to have influenced the submitted work.

\section{References}

1. Beere HM: "The stress of dying": the role of heat shock proteins in the regulation of apoptosis . J Cell Sci. 2004, 117:2641-51. 10.1242/jcs.01284

2. Zininga T, Ramatsui L, Shonhai A: Heat shock proteins as immunomodulants. Molecules. 2018, 23:2846. 10.3390/molecules23112846

3. Vihervaara A, Sistonen L: HSF1 at a glance . J Cell Sci. 2014, 127:261-6. 10.1242/jcs.132605

4. Zettervall S, Shuja F, Schermerhorn M: Primer in cerebrovascular disease: innominate and subclavian disease. Louis R. Caplan (ed): Elsevier, USA; 2017.

5. Mehmood R, McAlpine SR: Heat Shock Protein 27. Structure, Function, Cellular Role and Inhibitors. . Heat Shock Protein Inhibitors. 2015, 19:221-34. 10.1007/7355_2015_94

6. Vidyasagar A, Wilson NA, Djamali A: Heat shock protein 27 (HSP27): biomarker of disease and therapeutic target. Fibrogenesis Tissue Repair. 2012, 5:7. 10.1186/1755-1536-5-7

7. Den RB, Lu B: Heat shock protein 90 inhibition: rationale and clinical potential . Ther Adv Med Oncol. 2012, 4:211-8. 10.1177/1758834012445574

8. Jee H: Size dependent classification of heat shock proteins: a mini-review . J Exerc Rehabil. 2016, 12:255-9. 
9. Garrido C, Paul C, Seigneuric R, Kampinga HH: The small heat shock proteins family: the long forgotten chaperones. Int J Biochem Cell Biol. 2012, 44:1588-92. 10.1016/j.biocel.2012.02.022

10. Tóth M, Gombos I, Sántha M: Heat shock proteins and their role in human diseases . Acta Biologica Szegediensis. 2015, 59:121-41.

11. Akerfelt M, Morimoto RI, Sistonen L: Heat shock factors: integrators of cell stress, development and lifespan. Nat Rev Mol Cell Biol. 2010, 11:545-55. 10.1038/nrm2938

12. Neudegger T, Verghese J, Hayer-Hartl M, Hartl FU, Bracher A: Structure of human heat-shock transcription factor 1 in complex with DNA. Nat Struct Mol Biol. 2016, 23:140-6. 10.1038/nsmb.3149

13. Mazaira GI, Daneri-Becerra C, Zgajnar NR, Lotufo CM, Galigniana MD: Gene expression regulation by heatshock proteins: the cardinal roles of HSF1 and Hsp90. Biochem Soc Trans. 2018, 46:51-65. 10.1042/BST20170335

14. Trinklein ND, Chen WC, Kingston RE, Myers RM: Transcriptional regulation and binding of heat shock factor 1 and heat shock factor 2 to 32 human heat shock genes during thermal stress and differentiation. Cell Stress Chaperones. 2004, 9:21-8. 10.1379/481.1

15. Fujimoto M, Hayashida N, Katoh T, et al.: A novel mouse HSF3 has the potential to activate nonclassical heat-shock genes during heat shock. Mol Biol Cell. 2010, 21:106-16. 10.1091/mbc.e09-07-0639

16. Nakai A: Heat shock transcription factors and sensory placode development. BMB Rep. 2009, 42:631-5. 10.5483/bmbrep.2009.42.10.631

17. Macario AJL, de Macario EC, Cappello F: The Chaperonopathies-Diseases with Defective Molecular Chaperones. Springer, Netherlands; 2013. 10.1007/978-94-007-4667-1

18. Horváth I, Glatz A, Nakamoto H, et al.: Heat shock response in photosynthetic organisms: membrane and lipid connections. Prog Lipid Res. 2012, 51:208-20. 10.1016/j.plipres.2012.02.002

19. Balogh G, Péter M, Glatz A, et al.: Key role of lipids in heat stress management . FEBS Lett. 2013, 587:197080. 10.1016/j.febslet.2013.05.016

20. Sreedhar AS, Csermely P: Heat shock proteins in the regulation of apoptosis: new strategies in tumor therapy: a comprehensive review. Pharmacol Ther. 2004, 101:227-57. 10.1016/j.pharmthera.2003.11.004

21. Calderwood SK, Gong J: Heat shock proteins promote cancer: it's a protection racket . Trends Biochem Sci. 2016, 41:311-23. 10.1016/j.tibs.2016.01.003

22. Ciocca DR, Calderwood SK: Heat shock proteins in cancer: diagnostic, prognostic, predictive, and treatment implications. Cell Stress Chaperones. 2005, 10:86-103. 10.1379/csc-99r.1

23. Wei L, Liu TT, Wang HH, Hong HM, Yu AL, Feng HP, Chang WW: Hsp27 participates in the maintenance of breast cancer stem cells through regulation of epithelial-mesenchymal transition and nuclear factor- $\mathrm{kB}$. Breast Cancer Res. 2011, 13:R101. 10.1186/bcr3042 